genital infections was found in $11 \%$ of patients. $80 \%$ of the patients were in their genitally active period. $2 \%$ of the patients had multiple sexual partners. Pap smear results were distributed as follows: $8.4 \%$ normal, $53.6 \%$ non-specific inflammation, $14.8 \%$ specific HPV infection, 6\% atrophy, 4.2\% ASC-US (Atypical squamous cells of undetermined significance), 2.8\% ASC-H (Atypical squamous cells of undetermined significance-cannot exclude HSIL) ), 5.2\% LSIL (Lowgrade squamous intraepithelial lesion), 2\% AGUS (Atypical glandular cells of undetermined significance), $1.8 \%$ HSIL (High-grade squamous intraepithelial lesion), $0.6 \%$ detecting the presence of cancer cells.

Conclusion In Morocco, the establishment of a national mass cervical cancer screening program adapted to our epidemiological and socioeconomic context as well as the improvement of hygienic living conditions remains the best means to reduce the incidence and mortality related to cervical cancer

Disclosures The authors declare they have no conflict of interest.

\section{Quality of life after treatment}

\begin{tabular}{l|l}
\hline 44 & IMPACT OF CERVICAL CANCER ON QUALITY OF LIFE \\
AND SEXUAL FUNCTIONING OF FILIPINO PATIENTS \\
WHO UNDERWENT DEFINITIVE CHEMORADIATION IN \\
THE UNIVERSITY OF SANTO TOMAS HOSPITAL, MANILA, \\
PHILIPPINES
\end{tabular}

Erika Lourdes Adajar. University of Santo Tomas Hospital, Manila, Philippines

10.1136/ijgc-2020-ESGO.163

Impact of Cervical Cancer on Quality of Life and Sexual Functioning of Filipino Patients who Underwent Definitive Chemoradiation in the University of Santo Tomas Hospital, Manila, Philippines

Introduction/Background Cervical cancer is a serious health problem, with nearly 500,000 women developing the disease each year worldwide. The burden of disease of cervical cancer in the Philippines as a developing nation is high. Its incidence rate has persisted from the 1980s up to the present with an annual age-standardized rate of 22.5 cases per 100,000 women. Of the thousands of Filipino women who are diagnosed with cervical cancer, $56 \%$ will die within 5 years from the diagnosis.

The aim of this study was to determine the quality of life (QoL) and sexual functioning of Filipino patients with cervical cancer on first consult, 3 months, and 6 months of completion of definitive chemoradiation.

Methodology The study is a 2-year prospective longitudinal observational study. Patients were assessed for QoL and sexual functioning on first consult, 3 months, and 6 months of completion of chemoradiation using the European Organization for Research and Treatment of Cancer (EORTC) QoL Questionnaire QLQ-C30 and EORTC (QLQ-CX24), respectively.

Results Fifty five patients were included for the analysis, and the mean age at the time of the interview was 52 years. Six months after the definitive chemoradiation, patients showed improved global health status/QoL and better physical role, cognitive, and emotional functioning than first day of treatment. Patients updated lower recurrence of symptoms. As to the sexual functioning impact of definitive chemoradiation on patients with cervical cancer, the patients experienced more problems with sexual activity and sexual enjoyment. Moreover, it is reported that all sexual function scales are correlated with health status of patients 6 months after treatment.

Conclusion This paper aided the health care providers to have a better understanding of the QoL and sexual functioning of cervical cancer patients who deal with its treatment sequelae. In addition, this will help counsel cervical cancer patients on what they could expect in a long term since definitive chemoradiation will have a great impact on their QoL. Furthermore, this study will also contribute on how to improve further research for Filipino women with cervical cancer.

Disclosures This paper has no relevant financial or nonfinancial relationships to disclose.

\section{SEXUAL FUNCTION AFTER PELVIC RADIOTHERAPY: A BRIEF DESCRIPTIVE STUDY IN LOCALLY ADVANCED CERVICAL CANCER PATIENTS}

${ }^{1}$ Yosep Sutandar, ${ }^{2}$ Suzanna Patricia Mongan, ${ }^{2}$ Bismarck Joel Laihad, ${ }^{2}$ Frank Mitchell M Wagey. 'Department of Obstetrics and Gynecology, Faculty of Medicine Universitas Sam Ratulangi; ${ }^{2}$ Division of Gynecologic Oncology, Dept. Obstetrics and Gynecology, Faculty of Medicine Universitas Sam Ratulangi

\subsection{6/ijgc-2020-ESGO.164}

Introduction/Background Cervical cancer is the most common gynecological cancers in Indonesia, 74\% of all cases of gynecologic cancers. In 2018, the incidence of cervical cancer is 9.3\% in Indonesia, $84.6 \%$ were diagnosed as advanced stage. The primary treatment in advanced cervical cancer is radiotherapy, especially pelvic radiotherapy. Although pelvic radiotherapy is the modality of choice for treatment in advanced cervical cancer, but it has side effects that can affect woman's sexual function.

Methodology The subjects of this study were 34 patients who were diagnosed with cervical cancer stage IIB-IVA and underwent pelvic radiotherapy based on local hospital database in Gynecologic Outpatient Clinic in Kandou Hospital from September 1st 2019 to February 29th 2020. All patients were interviewed with Female Sexual Function Index (FSFI) questionnaire. This questionnaire measures five groups of questions, which are sexual desire, arousal, lubrication, orgasm, satisfaction, and pain to assess sexual function in women.

Results From 34 patients who have undergone pelvic radiotherapy, the FSFI scores of 32 patients are below 26.5 and categorized as female sexual dysfunction (FSD), while two patients have score more than 26.6. The mean of FSFI Score is 10.0 .

Conclusion Pelvic radiotherapy has negative effect on the vaginal mucosa. This effect has significantly reduced the sexual function in women after pelvic radiotherapy. Assessment of the Sexual Function of every patients who have undergone pelvic radiotherapy is important to improve comprehensive care for female cancer patients.

Disclosures The authors declare no conflict of interest, financial, or otherwise. 